\title{
Assistência de enfermagem aos pacientes com cardiotoxicidade induzidas por
} quimioterápicos

\author{
Nursing care in patients with cardiotoxicity induced by chemotherapy \\ Atención de enfermería en pacientes con cardiotoxicidad inducida por quimioterapia
}

\author{
Jonas Magno dos Santos Cesário \\ ORCID: https://orcid.org/0000-0003-1785-3555 \\ Faculdade Unyleya, Brasil \\ E-mail: prof.jonasmagno@unyleya.edu.br \\ Victor Hugo de Paula Flauzino \\ ORCID: https://orcid.org/0000-0001-5156-0030 \\ Faculdade Unyleya, Brasil \\ E-mail: prof.victorflauzino@unyleya.edu.br \\ Luana de Oliveira Hernandes \\ ORCID: https://orcid.org/0000-0001-8828-7146 \\ Associação Brasileira de Enfermeiros Cientistas, Brasil \\ E-mail: luanaoliveira2306@gmail.com \\ Daiana Moreira Gomes \\ ORCID: https://orcid.org/0000-0001-9387-0619 \\ Associação Brasileira de Enfermeiros Cientistas, Brasil \\ E-mail: daigomes_87@hotmail.com \\ Priscila Gramata da Silva Vitorino \\ ORCID: https://orcid.org/0000-0002-1201-6945 \\ Associação Brasileira de Enfermeiros Cientistas, Brasil \\ E-mail: prigramaenf@yahoo.com.br
}

\begin{abstract}
Resumo
A cardiotoxicidade induzida pelo uso de quimioterápicos pode gerar acometimentos cardíacos devastadores piores que o câncer, além de atrapalhar o tratamento e prognóstico do paciente. Este estudo teve como objetivo descrever os fatores de risco para a cardiotoxidade por quimioterápicos e, mais especificamente, descrever os cuidados de enfermagem no paciente que apresentou cardiotoxicidade por quimioterápicos e radioterapia. A pesquisa é uma revisão bibliográfica de abordagem descritiva e qualitativa. Foi realizada uma busca nos Bancos de dados do Google Acadêmico, BVS (biblioteca virtual em saúde) e SciELO (Scientific Electronic Library Online), que resultou em uma amostra final de 24 artigos. Após a análise, foi possível identificar que os principais fatores de risco para desenvolver a cardiotoxidade são: idade acima de 60 anos, hipertensão, cardiopatias e uso de radioterapia torácica. Mas antineoplásicos anticíclicos e o trastuzumabe, antraciclinas, alquilantes, anticorpos monoclonais e inibidores da tirosina quinase são desencadeadores de cardiotoxidade, no momento em que estas quimioterapias são empregadas no tratamento de câncer é necessário que se realize uma avaliação da função cardíaca antes e depois da administração deste quimioterápicos. Existe a possibilidade de tais medicamentos ocasionarem insuficiência cardíaca, isquemias, arritmia, peri-cardiopatias entre outros problemas caso ocorra a cardiotoxicidade. Os principais cuidados de enfermagem na cardiotoxidade induzida por quimioterápicos são: verificar sinais vitais, peso e altura; avaliar os resultados de exames; notificar o médico sobre complicações; intervir imediatamente na ocorrência de possíveis efeitos colaterais durante a medicação.
\end{abstract}

Palavras-chave: Cuidados de enfermagem; Anestesia; Oncologia cirúrgica.

\begin{abstract}
Cardiotoxicity induced using chemotherapeutic agents can generate devastating cardiac problems worse than cancer, in addition to hindering the patient's treatment and prognosis. This study aimed to describe the risk factors for cardiotoxicity by chemotherapy and, more specifically, to describe nursing care for patients who had cardiotoxicity due to chemotherapy and radiation therapy. The research is a bibliographic review with a descriptive and qualitative approach. A search was performed in the Google Scholar Databases, VHL (virtual health library) and SciELO (Scientific Electronic Library Online), which resulted in a final sample of 24 articles. After the analysis, it was possible to identify that the main risk factors for developing cardiotoxicity are age over 60 years, hypertension, heart disease and use of chest radiotherapy. But anticyclic antineoplastic agents and trastuzumab, anthracyclines, alkylating agents, monoclonal antibodies, and tyrosine kinase inhibitors are triggers of cardiotoxicity, the moment these chemotherapies are used in the treatment of cancer it is necessary to carry out an assessment of cardiac function before and after administration of this chemotherapy. There is a possibility that such drugs may cause heart failure,
\end{abstract}


ischemia, arrhythmia, peri-cardiopathies, among other problems if cardiotoxicity occurs. The main nursing care in chemotherapy-induced cardiotoxicity are checking vital signs, weight, and height; evaluate the results of exams; notify the doctor about complications; intervene immediately in the occurrence of possible side effects during medication.

Keywords: Cardiotoxicity; Oncology; Nursing care.

\section{Resumen}

La cardiotoxicidad inducida por el uso de agentes quimioterápicos puede generar problemas cardíacos devastadores peores que el cáncer, además de dificultar el tratamiento y el pronóstico del paciente. Este estudio tuvo como objetivo describir los factores de riesgo de cardiotoxicidad por quimioterapia y, más específicamente, describir los cuidados de enfermería para pacientes que presentaban cardiotoxicidad por quimioterapia y radioterapia. La investigación es una revisión bibliográfica con enfoque descriptivo y cualitativo. Se realizó una búsqueda en las Bases de Datos Google Scholar, BVS (biblioteca virtual de salud) y SciELO (Scientific Electronic Library Online), que resultó en una muestra final de 24 artículos. Tras el análisis se pudo identificar que los principales factores de riesgo para desarrollar cardiotoxicidad son: edad mayor de 60 años, hipertensión, cardiopatía y uso de radioterapia torácica. Pero los agentes antineoplásicos anticíclicos y trastuzumab, antraciclinas, agentes alquilantes, anticuerpos monoclonales e inhibidores de la tirosina quinasa son desencadenantes de cardiotoxicidad, en el momento en que estas quimioterapias se utilizan en el tratamiento del cáncer es necesario realizar una evaluación de la función cardíaca antes y después de la administración de esta quimioterapia. Existe la posibilidad de que dichos fármacos provoquen insuficiencia cardíaca, isquemia, arritmia, peri-cardiopatías, entre otros problemas si se presenta cardiotoxicidad. Los principales cuidados de enfermería en cardiotoxicidad inducida por quimioterapia son: control de signos vitales, peso y talla; evaluar los resultados de los exámenes; notificar al médico sobre las complicaciones; intervenir inmediatamente en la aparición de posibles efectos secundarios durante la medicación.

Palabras clave: Cardiotoxicidad; Oncología; Cuidados de enfermería.

\section{Introdução}

No Brasil há muitos pacientes com câncer, no qual representa cerca de 25\%. Em 2018, eram estimados 59.120 casos de tumores malignos. Os principais destaques são o câncer de próstata e os tumores de mama, que são uma das principais causas de morte para os brasileiros. Dados do Ministério da Saúde apontam que cerca de 25\% a 35\% dos pacientes recebem tratamento tardio em um hospital de referência, mas a quimioterapia é necessária devido à metástase, portanto, o encaminhamento é obrigatório. (Argani et al., 2018)

O uso da quimioterapia progrediu muito e reduziu a mortalidade relacionada ao câncer, com a recuperação de milhares de pessoas. No entanto, a maior sobrevivência não impediu que houvesse um aumento na incidência de efeitos colaterais, que foram bem relatados nos últimos anos, o que incluí a cardiotoxicidade por quimioterápicos, uma das principais causas das doenças cardiovasculares. (Gripp et al., 2018).

A cardiotoxicidade induzida pelo uso de quimioterápicos pode gerar acometimentos cardíacos devastadores piores que o câncer, além de atrapalhar o tratamento e prognóstico do paciente. A manifestação da cardiotoxicidade pode ocorrer de diferentes formas como lesões valvular, coronária ou pericárdica, arritmias, disfunção ventricular e insuficiência cardíaca (Almeida, 2016).

Os quimioterápicos como as antraciclinas (doxorrubicina, idarrubicina e epirrubicina), causam a cardiotoxidade conforme o tempo e a quantidade de dose cumulativa no organismo, ocasiona lesão em células cardíacas e no interstício, o que pode ocasionar disfunção sistólica tardia e a disfunção diastólica precoce (Maia et al., 2015)

O enfermeiro deve obter o conhecimento sobre o risco de cardiotoxidade induzida por agentes quimioterápicos para realizar uma assistência de enfermagem segura e com qualidade. A Implementação dos cuidados de enfermagem exige a pluralidade e expertise do enfermeiro para assistir o paciente em uso contínuo da quimioterapia, no qual é fundamental que o enfermeiro atue antes e depois da administração de quimioterápicos, além de observar os sinais e sintomas de cardiotoxidade, por meio disto surgi a seguinte pergunta: Como deve ser executada a assistência de enfermagem em pacientes que apresentam cardiotoxicidade por quimioterapia e radioterapia?

Devido a importância da temática e pela ausência de estudos na área, este estudo teve como objetivo descrever os 
fatores de risco para a cardiotoxidade por quimioterápicos e, mais especificamente, descrever os cuidados de enfermagem no paciente que apresentou cardiotoxicidade por quimioterápicos e radioterapia.

\section{Metodologia}

O estudo foi realizado por meio de uma revisão bibliográfica de abordagem descritiva e qualitativa, que de acordo com Cesário, Flauzino e Mejia (2020), fundamenta-se com base em material que já foram construídos, o que incluí artigos científicos publicados em periódicos acadêmicos. Inicialmente, buscou-se reunir evidências para responder a seguinte pergunta de pesquisa: como deve ser executada a assistência de enfermagem aos pacientes que apresentam cardiotoxicidade por quimioterapia e radioterapia?

No DeCS (Descritores em Ciências da Saúde), encontrou-se os seguintes descritores: Cardiotoxicidade, oncologia e cuidados de enfermagem. Os bancos de dados utilizados foram o Google Acadêmico, BVS (biblioteca virtual em saúde) e SciELO (Scientific Electronic Library Online). No Google Acadêmico utilizou-se cada um dos descritores entre aspas ("'). Na BVS (biblioteca virtual em saúde), foi utilizado a opção pesquisa avançada, selecionada as bases da BDENF (Banco de Dados em Enfermagem), LILACS (Literatura Latino-Americana e do Caribe em Ciências da Saúde) e o operador lógico booleano “OR" e "AND". Na SciELO foi utilizada a opção pesquisa avançada e o operador lógico booleano "OR" e "AND".

Estabeleceu-se como critérios de inclusão, artigos acadêmicos publicados entre 2015 e 2021, na língua portuguesa, disponíveis de forma gratuita e nos bancos de dados já mencionados, que respondessem à pergunta de pesquisa. Excluíram-se artigos repetidos encontrados nas bases de dados, resumos, artigos inferiores a 2015 e artigos que não respondiam o problema da pesquisa. A coleta dos dados foi realizada no mês de março, por 5 pesquisadores de forma independente. Os resultados das buscas pelos dados e do número final de publicações que irão compor a revisão serão apresentados por meio de um fluxograma elaborado por Peters et al., 2015, denominado fluxograma de Prisma, que tem o objetivo de melhorar a organização da busca por estudos, além de melhorar a consistência do relato da revisão sistemática. O fluxograma mostra como foi organizada as pesquisas realizadas nos Bancos de dados da BVS, ScIELO e Google escolar, na qual foi organizada em 4 etapas distintas compostas pelos critérios de exclusão e inclusão utilizados durante a pesquisa, conforme pode ser observado na Figura 1. 
Figura 1: Fluxograma de Prisma.

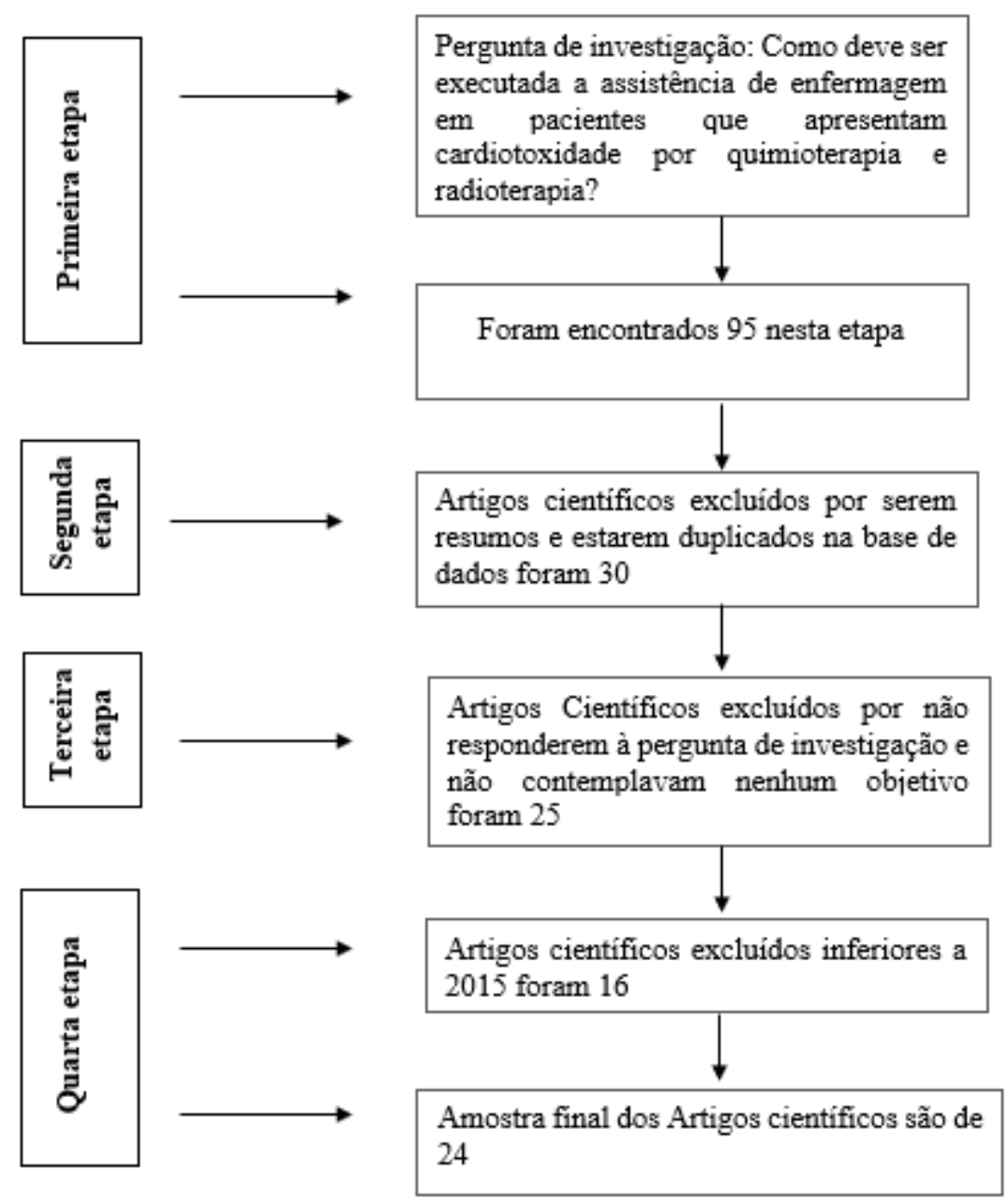

Fonte: Autores (2021).

\section{Resultados}

Os artigos científicos estão distribuídos nas bases de dados da BVS, Google Acadêmico e ScIELO, conforme mostra a Tabela 1 .

Tabela 1 - Resultados da busca nas bases de dados.

\begin{tabular}{c|c|c|c|c|c|c}
\hline \multicolumn{2}{c|}{ BVS } & \multicolumn{2}{c|}{ ScIELO } & \multicolumn{2}{c|}{ Google Acadêmico } & Amostra final \\
\hline Total & 20 & Total & 35 & Total & 40 & \multirow{2}{*}{24 artigos científicos } \\
\cline { 1 - 5 } Excluídos & 15 & Excluídos & 26 & Excluídos & 30 & \\
\hline Incluídos & 05 & Incluídos & 09 & Incluídos & 10 & \\
\hline
\end{tabular}

Fonte: Autores (2021).

Os dados que foram encontrados durante a pesquisa estão dispostos em duas categorias de acordo com a sua temática: Categoria A - Fatores de risco para a cardiotoxicidade em paciente com uso de quimioterápicos e Categoria B - Cuidados de enfermagem ao paciente com cardiotoxicidade quimioterápicos e radioterapia. O Quadro 1 apresenta os artigos de revisão da literatura que foram incluídos na categoria A, com as seguintes variáveis: autor/ano, título, objetivo e periódico. 
Quadro 1. Artigos incluídos na categoria temática A.

\begin{tabular}{|c|c|c|c|}
\hline Autor/ano & Título & Objetivo & Periódico \\
\hline $\begin{array}{l}\text { Guimarães et al., } \\
2015\end{array}$ & $\begin{array}{l}\text { Hiperatividade simpática } \\
\text { cardíaca após Quimioterapia: } \\
\text { sinal precoce de } \\
\text { cardiotoxicidade? }\end{array}$ & $\begin{array}{l}\text { Avaliar e comparar a presença de alterações } \\
\text { cardiovasculares entre pacientes com câncer de } \\
\text { mama submetidas à quimioterapia com } \\
\text { antracíclicos e trastuzumabe e apenas a } \\
\text { antracíclico. }\end{array}$ & $\begin{array}{l}\text { Arquivos brasileiros } \\
\text { de cardiologia }\end{array}$ \\
\hline Moura et al., 2015 & $\begin{array}{l}\text { Cardiotoxicidade induzida pela } \\
\text { doxorrubicina: } \\
\text { diagnóstico } \quad \text { e } \\
\text { antioxidantes }\end{array}$ & $\begin{array}{l}\text { Reunir informações acerca da patogenia, dos } \\
\text { métodos diagnósticos para lesões induzidas pela } \\
\text { DOX e das terapias antioxidantes utilizadas. }\end{array}$ & $\begin{array}{l}\text { Enciclopédia } \\
\text { Biosfera }\end{array}$ \\
\hline Almeida, 2016 & $\begin{array}{l}\text { Cardiotoxicidade subclínica em } \\
\text { oncologia: o impacto da } \\
\text { detecção precoce }\end{array}$ & $\begin{array}{l}\text { Avaliar prospectivamente a evolução da } \\
\text { contractilidade miocárdica, avaliada pela } \\
\text { deformação miocárdica longitudinal por speckle } \\
\text { tracking, numa população de considerável } \\
\text { dimensão, } 158 \text { doentes com neoplasia da mama } \\
\text { submetidas a quimioterapia com antraciclinas e } \\
\text { transtuzumab, e a sua relação com o } \\
\text { desenvolvimento de CT, estabelecido de acordo } \\
\text { com as recomendações }\end{array}$ & $\begin{array}{l}\text { Revista Portuguesa } \\
\text { de Cardiologia }\end{array}$ \\
\hline Maia et al., 2017 & $\begin{array}{l}\text { Cardiotoxicidade decorrente do } \\
\text { Tratamento com Doxorrubicina } \\
\text { e Exercício Físico: Revisão } \\
\text { Sistemática. }\end{array}$ & $\begin{array}{l}\text { Verificar na literatura se a prática de exercícios } \\
\text { possui um efeito protetor contra a toxicidade } \\
\text { cardíaca decorrente do tratamento com } \\
\text { doxorrubicina. }\end{array}$ & $\begin{array}{l}\text { International Journal } \\
\text { of Cardiovascular } \\
\text { Sciences. }\end{array}$ \\
\hline $\begin{array}{l}\text { Fassio \& } \\
\text { Delcastillo, } 2017\end{array}$ & $\begin{array}{l}\text { Aplicação da Ecocardiografia na } \\
\text { Detecção Precoce de } \\
\text { Cardiotoxicidade por } \\
\text { Quimioterapia }\end{array}$ & $\begin{array}{l}\text { Analisar a cardiotoxicidade produzida por } \\
\text { antraciclinas, anticorpos monoclonais e sua } \\
\text { associação através da indicação de alterações na } \\
\text { ecocardiografia convencional, por meio da fração } \\
\text { de ejeção do ventrículo esquerdo e strain } \\
\text { longitudinal global. }\end{array}$ & $\begin{array}{lr}\text { ABC }- & \text { Arquivos } \\
\text { Brasileiros } & \text { de } \\
\text { Cardiologia }\end{array}$ \\
\hline $\begin{array}{l}\text { Tavares et al., } \\
2017\end{array}$ & $\begin{array}{l}\text { Cardiotoxicidade associada à } \\
\text { terapia antineoplásica mamária } \\
\text { com trastuzumabe }\end{array}$ & $\begin{array}{l}\text { Avaliar artigos científicos que demonstraram } \\
\text { cardiotoxicidade associada à terapia } \\
\text { antineoplásica com trastuzumabe. Evidenciar a } \\
\text { importância da avaliação do sistema } \\
\text { cardiovascular previamente ao tratamento com o } \\
\text { anticorpo monoclonal trastuzumabe. }\end{array}$ & $\begin{array}{l}\text { Revista Científica do } \\
\text { ITPAC }\end{array}$ \\
\hline Gripp et al., 2018. & $\begin{array}{l}\text { Acurácia do Strain Longitudinal } \\
\text { Global na Predição de } \\
\text { Cardiotoxicidade em uma } \\
\text { Coorte de Pacientes com Câncer }\end{array}$ & $\begin{array}{l}\text { Avaliar a incidência de cardiotoxicidade entre } \\
\text { pacientes tratados para câncer de mama, os } \\
\text { fatores independentes associados a esse evento e } \\
\text { a capacidade do strain em identificá-lá }\end{array}$ & $\begin{array}{l}\text { Arquivos Brasileiros } \\
\text { de Cardiologia }\end{array}$ \\
\hline
\end{tabular}


Research, Society and Development, v. 10, n. 6, e34210615355, 2021

(CC BY 4.0) | ISSN 2525-3409 | DOI: http://dx.doi.org/10.33448/rsd-v10i6.15355

\begin{tabular}{|c|c|c|c|}
\hline & $\begin{array}{l}\text { de Mama em Tratamento com } \\
\text { Antracíclicos e/ou Trastuzumab }\end{array}$ & precocemente. & \\
\hline Argani et al., 2019 & $\begin{array}{l}\text { Avaliação da Cardiotoxicidade } \\
\text { induzida por Quimioterapia em } \\
\text { pacientes com Câncer de Mama }\end{array}$ & $\begin{array}{l}\text { Avaliar a alteração da função cardíaca em } \\
\text { pacientes com câncer de mama tratadas com } \\
\text { quimioterapia }\end{array}$ & $\begin{array}{l}\text { Clinical Oncology } \\
\text { Letters }\end{array}$ \\
\hline Barros et al., 2019 & $\begin{array}{l}\text { Alteração Contrátil Segmentar } \\
\text { Ventricular Esquerda é Preditor } \\
\text { Independente } \\
\text { Cardiotoxicidade em Pacientes } \\
\text { com Câncer de Mama em } \\
\text { Tratamento Quimioterápico }\end{array}$ & $\begin{array}{l}\text { Analisar a associação entre o surgimento de } \\
\text { alteração contrátil segmentar ventricular esquerda } \\
\text { (ACSVE) e } \quad \text { o desenvolvimento de } \\
\text { cardiotoxicidade em pacientes com câncer de } \\
\text { mama em tratamento com quimioterapia. }\end{array}$ & $\begin{array}{lr}\text { ABC }- & \text { Arquivos } \\
\text { Brasileiros } & \text { de } \\
\text { Cardiologia } & \end{array}$ \\
\hline $\begin{array}{l}\text { Fernandes et al., } \\
2019\end{array}$ & $\begin{array}{l}\text { Avaliação econômica do uso de } \\
\text { dexrazoxano na profilaxia, de } \\
\text { cardiotoxicidade em crianças em } \\
\text { tratamento quimioterápico com } \\
\text { antraciclinas. }\end{array}$ & $\begin{array}{l}\text { Realizar uma avaliação econômica e analisar o } \\
\text { impacto orçamentário da utilização do } \\
\text { dexrazoxano na prevenção de cardiotoxicidade } \\
\text { em pacientes pediátricos em uso de antraciclinas } \\
\text { no Sistema Único de Saúde (SUS). }\end{array}$ & $\begin{array}{l}\text { Caderno de saúde } \\
\text { pública. }\end{array}$ \\
\hline $\begin{array}{l}\text { Oliveira et al., } \\
2019\end{array}$ & $\begin{array}{l}\text { Paciente em tratamento de } \\
\text { neoplasia mamária sem } \\
\text { hiperexperssão her-2, evoluindo } \\
\text { com cardiotoxicidade, disfunção } \\
\text { ventricular esquerda } \\
\text { sintomática. }\end{array}$ & $\begin{array}{l}\text { Adoção de estratégias de prevenção, o } \\
\text { diagnóstico precoce e o tratamento das doenças } \\
\text { cardiovasculares. }\end{array}$ & $\begin{array}{l}\text { Revista Eletrônica } \\
\text { Acervo Saúde. }\end{array}$ \\
\hline Paula et al., 2019 & $\begin{array}{l}\text { Opções Contemporâneas de } \\
\text { Rastreio da Cardiotoxicidade } \\
\text { Relacionada a Tratamentos } \\
\text { Oncológicos. }\end{array}$ & $\begin{array}{l}\text { Discutidos os métodos clássicos e } \\
\text { contemporâneos no diagnóstico, rastreio e manejo } \\
\text { da cardiotoxicidade. }\end{array}$ & $\begin{array}{l}\text { Revista Brasileira de } \\
\text { Cancerologia. }\end{array}$ \\
\hline
\end{tabular}

Fonte: Autores (2021).

O Quadro 2 mostra os artigos de revisão da literatura que foram encontrados na categoria B, com as seguintes variáveis: Autor/ano, título, objetivo e periódico.

Quadro 2. Artigos incluídos na categoria temática B.

\begin{tabular}{|l|l|l|l|}
\hline \multicolumn{1}{|c|}{ Autor/ano } & \multicolumn{1}{|c|}{ Título } & \multicolumn{1}{c|}{ Objetivo } & \multicolumn{1}{c|}{ Periódico } \\
\hline $\begin{array}{l}\text { Guimarães et al., } \\
2015\end{array}$ & $\begin{array}{l}\text { Ações de enfermagem frente } \\
\text { às reações a quimioterápicos } \\
\text { em pacientes oncológicos }\end{array}$ & $\begin{array}{l}\text { Descrever as ações de enfermagem } \\
\text { frente às reações a quimioterápicos em } \\
\text { pacientes oncológicos. }\end{array}$ & $\begin{array}{l}\text { Revista de pesquisa cuidado é } \\
\text { fundamental online }\end{array}$ \\
\hline $\begin{array}{l}\text { Oliveira, Sobrinho, } \\
\text { Cunha, 2016 }\end{array}$ & $\begin{array}{l}\text { Manuseio da dor crônica em } \\
\text { pacientes oncológicos pela } \\
\text { equipe de enfermagem. }\end{array}$ & $\begin{array}{l}\text { identificar, na literatura, como a equipe } \\
\text { de enfermagem manuseia a dor crônica } \\
\text { nos pacientes oncológicos. }\end{array}$ & $\begin{array}{l}\text { Sociedade Brasileira para o da Dor. } \\
\text { Estudo }\end{array}$ \\
\hline
\end{tabular}




\begin{tabular}{|c|c|c|c|}
\hline Peiter et al., 2016 & $\begin{array}{l}\text { Gestão do cuidado de } \\
\text { enfermagem ao paciente } \\
\text { oncológico num hospital } \\
\text { geral: uma Teoria } \\
\text { Fundamentada nos Dados. }\end{array}$ & $\begin{array}{l}\text { Compreender o significado da gestão do } \\
\text { cuidado de enfermagem aos pacientes } \\
\text { oncológicos internados num hospital } \\
\text { geral. }\end{array}$ & $\begin{array}{l}\text { Referência Revista de } \\
\text { Enfermagem. }\end{array}$ \\
\hline Borges et al., 2018 & $\begin{array}{l}\text { Avaliação dos registros de } \\
\text { enfermagem acerca das } \\
\text { manifestações clínicas de } \\
\text { cardiotoxicidade }\end{array}$ & $\begin{array}{l}\text { Avaliar os registros de enfermagem em } \\
\text { um ambulatório de quimioterapia, } \\
\text { acerca das manifestações clínicas de } \\
\text { cardiotoxicidade e apresentar uma } \\
\text { proposta de roteiro de informações } \\
\text { direcionada aos registros dos cuidados } \\
\text { nessa clientela. }\end{array}$ & $\begin{array}{l}\text { Revista Enfermagem Atual In } \\
\text { Derme. }\end{array}$ \\
\hline Lins, Souza, 2018 & $\begin{array}{l}\text { Formação dos enfermeiros } \\
\text { para o cuidado em oncologia }\end{array}$ & $\begin{array}{l}\text { Analisar os aspectos relacionados à } \\
\text { formação dos enfermeiros residentes, às } \\
\text { dificuldades e facilidades para o cuidado } \\
\text { em oncologia. }\end{array}$ & Revista de Enfermagem UFPE \\
\hline Oliveira et al., 2019 & $\begin{array}{lr}\text { Cuidados de } & \text { enfermagem } \\
\text { para pacientes } & \text { oncológicos } \\
\text { neutropênicos: } & \text { scoping } \\
\text { review. } & \end{array}$ & $\begin{array}{l}\text { Identificar os cuidados de enfermagem } \\
\text { relativos aos fatores de risco para } \\
\text { neutropenia febril. }\end{array}$ & $\begin{array}{l}\text { Revista norte mineira de } \\
\text { enfermagem. }\end{array}$ \\
\hline $\begin{array}{l}\text { Oliveira, } \\
\text { Silva, } 2019\end{array}$ & $\begin{array}{l}\text { Cuidados de enfermagem ao } \\
\text { paciente oncológico portador } \\
\text { de cateter totalmente } \\
\text { implantado }\end{array}$ & $\begin{array}{l}\text { Identificar os cuidados de enfermagem, } \\
\text { prestados pelo enfermeiro, com o cateter } \\
\text { venoso central totalmente implantado, } \\
\text { em pacientes oncológicos }\end{array}$ & $\begin{array}{l}\text { Estudo descritivo, transversal } \\
\text { com abordagem quantitativa }\end{array}$ \\
\hline Souza et al., 2019 & $\begin{array}{l}\text { Cuidados de enfermagem ao } \\
\text { paciente oncológico em } \\
\text { tratamento quimioterápico } \\
\text { ambulatorial }\end{array}$ & $\begin{array}{l}\text { Descrever os estudos que abordam os } \\
\text { cuidados de enfermagem aos pacientes } \\
\text { oncológicos adultos em tratamento } \\
\text { quimioterápico sistêmico ambulatorial }\end{array}$ & Revisão de Literatura \\
\hline Souza et al., 2019 & $\begin{array}{l}\text { Cuidados de enfermagem ao } \\
\text { paciente oncológico em } \\
\text { tratamento quimioterápico } \\
\text { ambulatorial }\end{array}$ & $\begin{array}{l}\text { Descrever os estudos que abordam os } \\
\text { cuidados de enfermagem aos pacientes } \\
\text { oncológicos adultos em tratamento } \\
\text { quimioterápico sistêmico ambulatorial }\end{array}$ & Revisão de Literatura \\
\hline $\begin{array}{l}\text { Anacleto, } \\
\text { Cecchetto, Riegel, } \\
2020\end{array}$ & 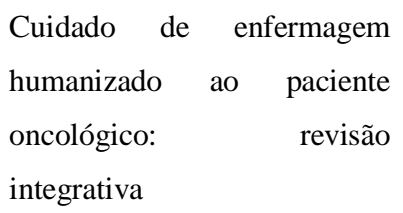 & $\begin{array}{l}\text { Verificar de que forma a equipe de } \\
\text { enfermagem promove o cuidado } \\
\text { humanizado ao paciente oncológico }\end{array}$ & Revisão Integrativa \\
\hline Silva, Pereira, 2020 & $\begin{array}{l}\text { A assistência à gestante com } \\
\text { câncer: o papel da equipe de } \\
\text { enfermagem }\end{array}$ & $\begin{array}{l}\text { Descrever qual é o papel do enfermeiro } \\
\text { diante da gestante com câncer. }\end{array}$ & $\begin{array}{l}\text { Revista JRG de estudos } \\
\text { acadêmicos }\end{array}$ \\
\hline
\end{tabular}




\begin{tabular}{|c|c|c|c|}
\hline Oliveira et al., 2020 & $\begin{array}{l}\text { Sistematização da Assistência } \\
\text { de Enfermagem: análise da } \\
\text { produção científica em } \\
\text { oncologia } \quad-\quad \text { revisão } \\
\text { integrativa. }\end{array}$ & $\begin{array}{l}\text { Analisar a produção científica em } \\
\text { oncologia acerca da SAE sob análise } \\
\text { das dificuldades enfrentadas pela } \\
\text { enfermagem para promover sua } \\
\text { implementação nas instituições de } \\
\text { saúde. }\end{array}$ & $\begin{array}{l}\text { Brazilian Journal of } \\
\text { Development. }\end{array}$ \\
\hline Celeste Maia, 2021 & $\begin{array}{l}\text { Cuidados de enfermagem } \\
\text { relacionados à } \\
\text { cardiotoxicidade envolvendo } \\
\text { drogas como a antraciclinas e } \\
\text { anticorpo monoclonais no } \\
\text { tratamento oncológico }\end{array}$ & $\begin{array}{l}\text { Realizar o embasamento teórico, } \\
\text { necessário para o manejo de pacientes } \\
\text { que estejam em uso desses devidos } \\
\text { antineoplásicos. }\end{array}$ & $\begin{array}{l}\text { Revisão bibliográfica do tipo } \\
\text { integrativo }\end{array}$ \\
\hline
\end{tabular}

Fonte: Autores (2021).

\section{Discussão}

\section{Categoria A - Fatores de risco para a cardiotoxicidade em paciente com uso de quimioterápicos}

No tratamento do câncer com quimioterapia, a cardiotoxicidade é uma reação adversa importante que pode levar a uma mortalidade considerável. Isso se deve a efeitos prejudiciais no sistema cardiovascular. Portanto, o monitoramento da função cardíaca desses pacientes torna-se crucial. O método diagnóstico mais utilizado para avaliar esses danos é a ecocardiografia, na qual os efeitos cardiotóxicos da quimioterapia podem ser confirmados. Além disso, apenas uma pequena porcentagem de pacientes é monitorada de forma adequada. (Argani et al., 2019).

No início do tratamento do câncer é realizada a avaliação do desempenho cardíaco, sabendo -se que no período do tratamento com antineoplásicos podem desencadear problemas cardiovasculares e cardiotoxicidade. Avaliação dos danos ocasionados são irreversíveis, com isso são avaliados dosagem medicamentosa e o tempo de tratamento específico para cada situação. Os problemas de cardiotoxicidade apresentam-se em médio a longo prazo no tratamento, mantendo avaliações contínuas da função cardiovascular (Oliveira et al., 2019)

$\mathrm{O}$ tratamento quimioterápico, principalmente em pacientes idosos, apresenta grande incidência de cardiotoxicidade. Esta se caracteriza por provocar danos celulares com possibilidade de reversão ou não, que condiz com à insuficiência cardíaca. Por isso o diagnóstico precoce por meio de métodos de imagem, como a ecocardiografia convencional, um exame de baixo custo, fácil acesso e com boa reprodutibilidade, tem diminuído significativamente a mortalidade de pacientes com câncer, em especial pacientes com câncer de mama. As atuais diretrizes recomendam a utilização do strain longitudinal global, que avalia a função global e ventricular esquerda, possibilita o tratamento precoce do dano do músculo cardíaco e contribuir para reduzir ou suspender o tratamento com quimioterápicos (Fassio \& Del Castillo, 2017).

Maia et al. (2017), avaliou que os fatores de risco para cardiotoxicidade estão relacionados ao uso de antineoplásicos que são utilizados no tratamento do câncer. Os problemas cardiovasculares que se apresentam após o uso deste componente da quimioterapia são irreversíveis em médio a longo prazo. A utilização de medicação com mecanismo cardioprotetores, com a perspectiva de diminuir o dano cardiovascular causado pela quimioterapia.

Os anticíclicos e o trastuzumabe são tipos de fármacos utilizados para seções de quimioterapia, que tem como objetivo o tratamento do câncer, porém, o seu uso pode causar cardiotoxicidade. A associação de anticíclicos e o trastuzumabe pode causar danos ainda maiores e proporcionar o risco de hiperatividade cardíaca, o que pode ser evitado ao intercalar as 
doses, pois há uma diminuição da cardiotoxicidade se não houver interação desses quimioterápicos. A cardiotoxicidade é desenvolvida de forma silenciosa e pode causar danos irreversíveis ao coração (Guimarães et al., 2015)

Conforme Paula et al. (2019), o fator de risco mais elevado para desenvolver problemas cardiovasculares é o uso de terapia com antineoplásicos, pois podem levar a cardiotoxicidade em alguns casos. As disfunções cardíacas podem estar relacionadas a tipos determinados de antineoplásicos como antraciclinas, alquilantes, anticorpos monoclonais e inibidores da tirosina quinase, que podem ser um desencadeador para problemas cardiovasculares moderados a graves, com uso em médio e longo prazo. A incidência de cardiotoxicidade apresentou elevação com uso destes antineoplásicos e por isso os autores deste estudo recomendaram a avaliação da função do músculo cardíaco antes e após a administração dos antineoplásicos, para que seja possível avaliar se existe cardiotoxicidade e se está afeta o coração de alguma forma.

As drogas quimioterápicas antraciclinas e anticorpos monoclonais humanizados são amplamente utilizados e eficazes no tratamento do câncer. No entanto, as antraciclinas podem induzir efeitos cardiotóxicos e o uso de trastuzumabe pode exacerbar a gravidade dessas reações adversas. Dentre eles, as alterações sistólicas segmentares observadas são um preditor independente de cardiotoxicidade, e se tornou uma ferramenta útil para avaliar pacientes em risco de CTRCT, que pode diagnosticar disfunção miocárdica precocemente e pode reduzir a morbimortalidade desses pacientes (Barros et al., 2019).

Segundo Fernandes et al. (2019) o uso prolongado de quimioterápicos como as antraciclinas podem desencadear cardiotoxicidade, os problemas cardiovasculares aparecem em médio a longo prazo, no qual ocasiona insuficiência cardíaca, arritmia, isquemias, Peri cardiopatias entre outros e os danos clínicos. Foi avaliado que o público infantil que realiza tratamento de câncer com as antraciclinas apresentou grande vulnerabilidade para desenvolver problemas cardíacos.

Para Gripp, et al. (2018) Embora bons resultados tenham sido alcançados com medicamentos antitumorais (antraciclinas e trastuzumabe) no tratamento do câncer, ainda é possível encontrar efeitos colaterais, entre eles a cardiotoxicidade, que é a causa do aumento da mortalidade. Identificar pacientes com alto risco de desenvolver cardiotoxicidade seria uma estratégia ideal para reduzir a mortalidade. Portanto, a análise do strain longitudinal total (SLG) após o tratamento com ácido antracenocarboxílico é o melhor parâmetro para detectar o risco de cardiotoxicidade. Observou-se que pacientes com diagnóstico de câncer sem tratamento antitumoral prévio e sem disfunção ventricular receberão o uso de antraciclinas e / ou trastuzumabe. O estiramento longitudinal global pode identificar precocemente pacientes que podem desenvolver cardiotoxicidade associada a antraciclinas e / ou trastuzumabe.

O fator de risco para a cardiotoxicidade é o uso da quimioterapia que tem como composto a antraciclinas, para diminuir os problemas cardíacos recomenda-se o uso de dexrazoxano de forma profilática em associação direta com as antraciclinas, no qual atua na redução do desenvolvimento de cardiotoxicidade e não influencia diretamente no aparecimento de tumor secundário (Fernandes et al., 2019).

É fato que o tratamento oncológico desencadeia inúmeros problemas cardíacos como efeito colateral. O desenvolvimento das drogas torna-se cada vez mais eficazes para o tratamento, com significativos poderes de cura, intensifica ainda mais o problema de cardiotoxicidade. No entanto, pacientes submetidas ao tratamento do câncer de mama, têm sido alvo da cardiotoxicidade devido aos fármacos utilizados para esse tipo de tratamento oncológico. A detecção precoce do problema, por meio do acompanhamento do paciente, é o ponto chave para impedir problemas maiores e assim diminuir os riscos de complicações futuras (Almeida, 2016).

\section{Categoria B - Cuidados de enfermagem ao paciente com cardiotoxicidade quimioterápicos e radioterapia}

Conforme Silva e Pereira (2020), o tratamento atual do câncer baseia-se na combinação de quimioterapia, radioterapia e cirurgia. Porém, a doença cardiovascular pode aparecer antecipadamente ou ser agravada na utilização de qualquer uma das opções convencionais de tratamento para o câncer. Apesar de as antraciclinas serem um dos agentes mais ativos no tratamento, 
apresenta forte tendência a causar cardiotoxicidade. Portanto, é de extrema importância conhecer a farmacocinética e farmacodinâmica dessas drogas, que irão influenciar diretamente na avaliação e controle dos efeitos colaterais sofridos pelo paciente em tratamento quimioterápico. É imprescindível que a equipe de enfermagem atue em todos os estágios da quimioterapia e instruir os pacientes e familiares, com a finalidade de elaborar um plano assistencial para uma assistência à saúde eficaz e de qualidade ao paciente (Celeste \& Maia, 2021).

Durante gestão da assistência de enfermagem o enfermeiro deve realizar estratégias, para identificar precocemente os sinais e sintomas de uma possível cardiotoxidade induzida por quimioterapia, pois o paciente se descompensa muito rápido e pode necessitar de cuidados intensivos. A equipe deve ser treinada e qualificada para prestar os cuidados de enfermagem durante o episódio de cardiotoxidade (Peiter et al., 2016).

Os principais sintomas de uma cardiotoxidade são: náuseas, vômitos e diarreia, fadiga, diminuição da massa muscular, aumento da atividade inflamatória, anemia, e alterações na cascata de coagulação. O enfermeiro também precisa estar atento ao grau de hidratação desses pacientes, pois a desidratação é uma das principais consequências. Manter a integridade da pele também é apontado como um dos objetivos do cuidado de enfermagem, visto que todas essas alterações podem alterar a integridade tissular. Uma avaliação cuidadosa do enfermeiro pode identificar essas potencialidades e elaborar um plano de cuidados para prevenir ou minimizar os efeitos causados pela cardiotoxidade ao paciente (Guimarães et al., 2015).

Os cuidados de enfermagem em pacientes que apresentam sinais de cardiotoxicidade são: verificar sinais vitais, peso e altura; avaliar os resultados de exames; notificar o médico sobre complicações; intervir imediatamente na ocorrência de possíveis efeitos colaterais durante a medicação, bem como realizar o registro de enfermagem completo e intercorrências do processo de enfermagem forma clara e concisa, a fim de proporcionar clareza a quem está lendo, garantir, assim, a continuidade da assistência de enfermagem. Itens que são indispensáveis nestes registros são a identificação do paciente com o máximo de detalhes, o protocolo quimioterápico adotado, comorbidades e sinais vitais, medicações de uso contínuo, especificação dos cateteres utilizados, descrição dos sintomas cardiotóxicos apresentados e manifestações gastrointestinais (Borges et al., 2018).

Para Oliveira et al. (2019), os pacientes que são submetidos a uso de quimioterapia e desenvolvem cardiotoxidade apresentam risco elevado de desenvolvimento de infecções devido a imunidade debilidade e sem defesa, o enfermeiro desempenha papel primordial na abordagem de sinais e sintomas relacionados a risco de estabilidade hemodinâmica e sepse. Os principais cuidados de enfermagem são: prevenção de sepse, orientações sobre autocuidado, consultas periódicas com enfermeira, higiene das mãos, cuidado com dispositivo venoso (cateter), orientar sobre fatores e sinais de risco de infecção e sepse, cuidados e avaliações nutricional (Oliveira, Fontes \& Silva, 2019).

Os pacientes que adquirem cardiotoxidade por quimioterapia relata bastante dor, a equipe de enfermagem pode auxiliar no manejo para o alívio da dor realiza método não farmacológico sendo, massagem terapêutica, apoio espiritual, medidas de conforto. Forma farmacológica e administração de medicação para controle álgico conforme escala de classificação para analgesia até uso de opioides para melhor manejo da dor em oncologia, onde equipe de enfermagem tem de avaliar os sinais vitais e de instabilidade hemodinâmica do paciente, juntamente a classificação e manejo adequado da dor (Oliveira, Sobrinho \& Cunha, 2016).

O enfermeiro que realiza as ações da assistência de enfermagem, para ser utilizada no direcionamento do cuidado ao paciente com base na sua observação, avaliação e de forma científica. O diagnóstico de cardiotoxidade induzida por quimioterápicos envolve alta complexidade no cuidado, diante deste obtemos responsabilidades e metas assistenciais para possibilitar e favorecer qualidade durante o tratamento, pois a cardiotoxicidade implica diretamente na vida do paciente no decorrer do processo de saúde-doença, ocasiona limitações da vida diária, ônus financeiro gerado em sua vida devido aos 
problemas de saúde, mudança na rotina de vida, alterações de ambientes, angustia, afastamento domiciliar, e alterações na autoimagem, sinais e sintomas e a dor (Oliveira et al., 2020).

O tratamento do paciente com cardiotoxidade induzida por quimioterápicos exige da equipe de enfermagem um acompanhamento especializado, assim abordagens clínicas que levem em consideração aspectos biopsicosocialespirituais, por meio da flexibilização na atuação e pluralidade de conhecimento, que estão atrelados a uma sistematização da assistência de qualidade são fatores cruciais para uma gerência de boa qualificação. É de responsabilidade do enfermeiro do setor de oncologia administrar os agentes quimioterápicos, trabalhar na elaboração de protocolos para prevenção, tratamento e redução de efeitos colaterais nos pacientes em uso de quimioterapia, além da listagem de ações de prevenção de riscos e agravos, por meio da educação dos pacientes e familiares, o que contribuirá para uma maior sobrevida do paciente (Souza et al., 2019).

Os profissionais de enfermagem precisam estar preparados e capacitados para oferecer um cuidado humanizado ao paciente oncológico com cardiotoxicidade, visto que a cardiotoxicidade induzida por quimioterapia deixa o paciente bastante fragilizado para desenvolver a sua rotina diária. A Política Nacional de Humanização está baseada em direcionar e implementar as melhores práticas de humanização da assistência no contexto do processo saúde-doença do paciente com neoplasias que desenvolveram problemas cardíacos relacionados ao uso de radioterapia ou quimioterapia (Anacleto, Cecchetto, Riegel, 2020).

\section{Considerações Finais}

No momento em que ocorre a cardiotoxicidade por quimioterápico ocorre efeitos deve a efeitos lesivos gerados no sistema cardiovascular. Durante o uso da quimioterapia com antineoplásico é avaliado a dose e o tempo de tratamento para cada caso, pois os problemas com a cardiotoxicidade apresentam-se em médio a longo prazo. por isso os autores deste estudo recomendaram a avaliação da função do músculo cardíaco antes e após a administração dos antineoplásicos, para que seja possível avaliar se existe cardiotoxicidade e se está afeta o coração de alguma forma.

Alguns fatores que podem contribuir para a cardiotoxicidade são: idade acima de 60 anos, hipertensão, cardiopatias e uso de radioterapia torácica. Mas antineoplásicos anticíclicos e o trastuzumabe, antraciclinas, alquilantes, anticorpos monoclonais e inibidores da tirosina quinase, são desencadeadores de cardiotoxicidade. O emprego destas drogas no tratamento de câncer, deve ser feita uma avaliação da função cardíaca antes da administração e após seu uso, pois ocasiona insuficiência cardíaca, isquemias, arritmia, peri-cardiopatias entre outros e os danos clínicos.

O enfermeiro deve ter conhecimento sobre os fatores de risco para a cardiotoxidade induzida por quimioterápico com a finalidade de realizar um plano de cuidados para identificar precocemente os sinais e sintomas, além de treinar a equipe de enfermagem para identificar sinais e sintomas como: náuseas, vômitos e diarreia, fadiga, diminuição da massa muscular, aumento da atividade inflamatória, anemia, e alterações na cascata de coagulação.

Os principais cuidados de enfermagem na cardiotoxidade é verificar sinais vitais, peso e altura; avaliar os resultados de exames; notificar o médico sobre complicações; intervir imediatamente na ocorrência de possíveis efeitos colaterais durante a medicação. O enfermeiro desempena um papel de extrema importância para prestar uma assistência de enfermagem de qualidade para a prevenção de infecção, educação sobre autocuidado, avaliações nutricionais e controle da dor, sempre deve ser levado em consideração os aspectos biopsicosocialespirituais, com práticas de humanização durante toda a assistência de enfermagem.

Após a elaboração desta pesquisa os autores esperam contribuir para o desenvolvimento de novas pesquisas que façam interface entre as áreas de cardiologia e oncologia, pois apesar dessas áreas exigirem atualização constante dos profissionais de saúde separadamente, em relação a convergência dos estudos ainda pouca produção acadêmica nacional. 


\section{Referências}

Almeida, A. G. (2017). Cardiotoxicidade subclínica em oncologia: o impacto da detecção precoce. Revista Portuguesa de Cardiologia. 36(1). 17-19. http://dx.doi.org/10.1016/j.repc.2016.06.009.

Anacleto, G.; Cecchetto, F. H. \& Riegel, F. (2020). Cuidado de enfermagem humanizado ao paciente oncológico: revisão integrativa. Revista Enfermagem Contemporânea, 9(2). 246-254. http://dx.doi.org/10.17267/2317-3378rec.v9i2.2737.

Argani, I. L et al. (2019) Avaliação da Cardiotoxicidade induzida por Quimioterapia em pacientes com Câncer de Mama. Clinical Oncology Letters. 3(1-2). 37-44. http://dx.doi.org/10.4322/col.2018.002.

Barros, M. V. L et al. (2019). Alteração Contrátil Segmentar Ventricular Esquerda é Preditor Independente de Cardiotoxicidade em Pacientes com Câncer de Mama em Tratamento Quimioterápico. Arquivos Brasileiros de Cardiologia, 112(1). 50-56. http://www.dx.doi.org/10.5935/abc.20180220.

Borges, M. D et al. (2018). Avaliação dos registros de enfermagem acerca das manifestações clínicas de cardiotoxicidade. Revista Enfermagem Atual In Derme. 86(24). 78. https://doi.org/10.31011/reaid-2018-v.86-n.24-art.78.

Celeste, L. E. N. \& Maia, M. R. (2021). Cuidados de enfermagem relacionados à cardiotoxicidade envolvendo drogas como a antraciclinas e anticorpo monoclonais no tratamento oncológico. Revista Pubsaúde, 5(95). https://dx.doi.org/10.31533/pubsaude5.a095.

Cesário, J. M. S.; Flauzino, V. H. P. \& Mejia, J. V. C. (2020). Metodologia científica: Principais tipos de pesquisas e suas caraterísticas. Revista Científica Multidisciplinar Núcleo do Conhecimento. 5(11). 23-33. 10.32749/nucleodoconhecimento.com.br/educacao/tipos-de-pesquisas.

Fassio, R. C. C. \& Del Castillo, J. M. (2017). Aplicação da Ecocardiografia na Detecção Precoce de Cardiotoxicidade por Quimioterapia. ABC. Imagem cardiovascular. (8)1. 12. 10.5935/2318-8219.20170004.

Fernandes, R. R. A, et al. (2019) Avaliação econômica do uso de dexrazoxano na profilaxia, de cardiotoxicidade em crianças em tratamento quimioterápico com antraciclinas. Caderno de saúde pública. 35(9) e00191518, 10.1590/0102-311X00191518.

Gripp, E. A. et al. (2018). Acurácia do Strain Longitudinal Global na Predição de Cardiotoxicidade em uma Coorte de Pacientes com Câncer de Mama em Tratamento com Antracíclicos e/ou Trastuzumab. Arquivos Brasileiros de Cardiologia. 1(1). 140-150. https://doi.org/10.5935/abc.20180021.

Guimarães, R. C. R. et al. (2015) Ações de enfermagem frente às reações a quimioterápicos em pacientes oncológicos. Revista de pesquisa cuidado é fundamental online. 7(2). 2440-2452. 10.9789/2175-5361.2015.v7i2.2440-2452.

Guimarães, S. P. M. M et al. (2015). Hiperatividade Simpática Cardíaca Após Quimioterapia: Sinal Precoce de Cardiotoxicidade? Arquivo Brasileiro de Cardiologia. 105(3). 228-234. http://dx.doi.org/10.5935/abc.20150075.

Lins, F. G. \& Souza, S. R. (2018). Formação dos enfermeiros para o cuidado em oncologia. Revista de Enfermagem UFPE. 12 (1). 66-74. https://doi.org/10.5205/1981-8963-v12i01a22652p66-74-2018.

Moura, L. R et al. (2015). Cardiotoxicidade induzida pela doxorrubicina: patogenia, diagnóstico e terapias antioxidantes. Enciclopédia Biosfera. (11)22. 711736. http://dx.doi.org/10.18677/Enciclopedia_Biosfera_2015_113.

Maia, T. N, et al. (2017). Cardiotoxicidade Decorrente do Tratamento com Doxorrubicina e Exercício Físico: Revisão Sistemática. International Journal of Cardiovascular Sciences. 30(1):70-80. https://doi.org/10.5935/2359-4802.20170004.

Oliveira, A. L.; Sobrinho, N. P. \& Cunha, B. A. S. (2016), Manuseio da dor crônica em pacientes oncológicos pela equipe de enfermagem. Sociedade Brasileira para o Estudo da Dor. 17(3). 219-22. DOI 10.5935/1806-0013.20160075.

Oliveira, D. A. L.; Fontes, R. A. \& Silva, M. B. (2019). Cuidados de enfermagem ao paciente oncológico portador de cateter totalmente implantado. VITTALLE-Revista de Ciências da Saúde. 31(1). 52-60.

Oliveira, P. P. et al. (2019). Cuidados de enfermagem para pacientes oncológicos neutropênicos: scoping review. Revista norte mineira de enfermagem. 8(2). 17-28. 10.5935/1806-0013.20160075.

Oliveira, P. R. N. L et al. (2019). Paciente em tratamento de neoplasia mamária sem hiperexperssão her-2, evoluindo com cardiotoxicidade, disfunção ventricular esquerda sintomática. Revista Eletrônica Acervo Saúde. 20. e612. https://doi.org/10.25248/reas.e612.2019.

Oliveira, T. R. et al. (2020) Sistematização da Assistência de Enfermagem: análise da produção científica em oncologia - revisão integrativa. Brazilian Journal of Development. 6(2). 9541-9555. 10.34117/bjdv6n2-314.

Paula, B. H. R. et al. (2019). Opções Contemporâneas de Rastreio da Cardiotoxicidade Relacionada a Tratamentos Oncológicos. Revista Brasileira de Cancerologia. 65(3). e-07388. https://doi.org/10.32635/2176-9745.RBC.2019v65n3.388.

Peiter, C. C et al. (2016). Gestão do cuidado de enfermagem ao paciente oncológico num hospital geral: uma Teoria Fundamentada nos Dados. ReferênciaRevista de Enfermagem. 4(11). 61-69.

Peters, M. D et al. (2015). Guidance for conducting systematic scoping reviews. International Journal of Evidence-Based Healthcare. 13(3). 141-146. 10.1097/XEB.0000000000000050.

Silva, D. P. \& Pereira, M. C. (2020). A assistência à gestante com câncer: o papel da equipe de enfermagem. Revista JRG de Estudos Acadêmicos. 3(6). 199216. http://doi.org/10.5281/zenodo.3891982

Souza, F. S. L, et al. (2019) Cuidados de enfermagem ao paciente oncológico em tratamento quimioterápico ambulatorial. Revista Eletrônica Acervo Saúde, (1)31. 838-838. https://doi.org/10.25248/reas.e838.2019.

Tavares, P. H. N, et al. (2017). Cardiotoxicidade associada à terapia antineoplásica mamária com trastuzumabe. Revista Científica do ITPAC. $10(1)$. 46-57. 